PROCEEDINGS OF THE

AMERICAN MATHEMATICAL SOCIETY

Volume 130, Number 1, Pages 283-291

S 0002-9939(01)06051-8

Article electronically published on May 25, 2001

\title{
NORMALITY AND DENSE SUBSPACES
}

\author{
A. V. ARHANGEL'SKII
}

(Communicated by Alan Dow)

\begin{abstract}
In the first section of this paper, using certain powerful results in $C_{p}$-theory, we show that there exists a nice linear topological space $X$ of weight $\omega_{1}$ such that no dense subspace of $X$ is normal. In the second and third sections a natural generalization of normality, called dense normality, is considered. In particular, it is shown in section 2 that the space $R^{c}$ is not normal on some countable dense subspace of it, while it is normal on some other dense subspace. An example of a Tychonoff space $X$, which is not densely normal on a dense separable metrizable subspace, is constructed. In section 3, a link between dense normality and relative countable compactness is established. In section 4 the result of section 1 is extended to densely normal spaces.
\end{abstract}

\section{INTRODUCTION}

Many Tychonoff spaces are not normal. This can happen even in the case of rather standard topological spaces; even a linear topological space need not be normal. To see this, it is enough to refer to the product of uncountably many copies of the space of real numbers. It is natural to look for some trace of normality in Tychonoff spaces, to analyze when they satisfy some weaker forms of normality. One of the most obvious conditions of this type is the existence of a dense normal subspace. Less obvious, more delicate normality type conditions can be formulated in terms of the existense of a dense subspace of a space $X$, which is normal, in some sense, with respect to $X$. In the first section of this paper we consider the following question: Is there a dense normal subspace in any Tychonoff space? This question seems to have been around for some time, although I have never seen it in print. An answer to this question is obtained as an application of $C_{p}$-theory, and is based on two beautiful theorems of D.P. Baturov [5], 6] and yet another result in $C_{p}$-theory.

In the second section of the paper we consider a generalization of normality, called dense normality. The notion of dense normality (see [2]) is based on one of the most interesting versions of relative normality: the concept of normality of a space on its subspace. It is shown that the space $R^{c}$ is not normal on some countable dense subspace of it, while it is normal on some other dense subspace. An example of a Tychonoff space $X$ which is not normal on a dense separable

Received by the editors July 13, 1998 and, in revised form, June 6, 2000.

1991 Mathematics Subject Classification. Primary 54C35, 54D15, 54D20.

Key words and phrases. Normal space, extent, Lindelöf number, Souslin number, $C_{p}$-theory, densely normal, $\kappa$-normal, $X$ normal on $Y, A$ concentrated on $Y$, pseudocompact, relative countable compactness, locally connected. 
metrizable subspace is constructed. In section 3 a link between dense normality and relative countable compactness is established. This leads to some interesting questions. In section 4 we blend together the main ideas of sections 1, 2 and 3.

By $C_{p}(X)$ we denote the space of continuous real-valued functions on a Tychonoff space $X$ in the topology of pointwise convergence. All spaces under consideration are assumed to be Tychonoff; $R$ stands for the space of real numbers, with the usual topology, $c=2^{\omega}$. Closures of open sets are called canonical closed sets.

\section{On the EXIstence of DENSE NORMal SUbSPaCES}

Theorem 1.1. The space $C_{p}\left(\omega_{1}+1\right)$ does not contain a dense normal subspace.

Proof. Assume the contrary, and let $Y$ be a dense normal subspace of $C_{p}\left(\omega_{1}+1\right)$. Then $Y$ is homeomorphic to a dense subspace of $R^{\omega_{1}}$ [1]. Now we have to refer to the following special case of a theorem of Baturov [6]:

Theorem 1.2. If $Y$ is a dense normal subspace of $R^{\omega_{1}}$, then every closed discrete subspace of $Y$ is countable (that is, the extent $e(Y)$ of $Y$ is countable).

By Theorem 1.2, the extent of $Y$ is countable. Now we need another result of D. Baturov [5] (see also [1]):

Theorem 1.3. If $X$ is a compact space, then for any subspace $Y$ of $C_{p}(X)$ the extent of $Y$ coincides with the Lindelöf number of $Y$.

By Theorem 1.3, $Y$ is Lindelöf. Since $Y$ is dense in $C_{p}\left(\omega_{1}+1\right)$, the set $Y$ of functions on $\omega_{1}+1$ separates points of $\omega_{1}+1$. On the other hand, it was shown in [4] that no Lindelöf subspace of $C_{p}\left(\omega_{1}+1\right)$ separates points of $\omega_{1}+1$ (see also [1]). This contradiction completes the proof of Theorem 1.1.

Notice that the space $X=C_{p}\left(\omega_{1}+1\right)$ has the smallest possible weight $\omega_{1}$ which can have a non-metrizable space. It also has some other nice properties: for example, it is monolithic, stable (see [1]), and has countable tightness [1]. It is also $\kappa$-normal 1]. On the other hand, it is not Dieudonné complete [1]. It turns out that a slight modification of the above construction will produce a realcompact space without dense normal subspace. Recall that a space $X$ is submetrizable if it can be mapped by a one-to-one continuous mapping onto a metrizable space. Notice that every submetrizable space with countable Souslin number is hereditarily realcompact [7] and has the diagonal $G_{\delta}$.

Theorem 1.4. There exists a submetrizable space $X$ with countable Souslin number such that no dense subspace of $X$ is normal.

Proof. The space $\omega_{1}+1$ is a subspace of the Tychonoff cube $I^{\omega_{1}}$. The space $I^{\omega_{1}}$ is separable, therefore, we can fix a countable dense subspace $A$ of $I^{\omega_{1}}$. Put $K=A \cup\left(\omega_{1}+1\right)$. Then $K$ is a $\sigma$-compact separable space of the cardinality $\omega_{1}$, and $\omega_{1}+1$ is a closed subspace of it. The space $X=C_{p}(K)$ does not contain a dense Lindelöf subspace, since otherwise $C_{p}\left(\omega_{1}+1\right)$ would have contained such a subspace, which is not the case. Now arguing exactly as in the proof of Theorem 1.1 (notice that Theorem 1.3 remains true in the class of $\sigma$-compact spaces), we arrive at the conclusion that no dense subspace of $X$ is normal. On the other hand, since $K$ is separable, there is a natural one-to-one continuous mapping of $X$ onto a separable metrizable space (the restriction mapping). Therefore, $X$ is hereditarily realcompact, and all points in $Z$ are $G_{\delta}$ 's. Notice that the weight of $X$ is $\omega_{1}$. 
Question 1. Does there exist a first countable Tychonoff space $X$ such that no dense subspace of $X$ is normal?

\section{ON NORMALITY OF SPACES ON DENSE SUBSPACES}

One of the main results of this section, Theorem 2.7, establishes that $R^{c}$ can be not normal on a countable dense subspace of it, though $R^{c}$ is densely normal.

Recall that a space $X$ is $\kappa$-normal if every two disjoint canonical closed subsets of $X$ can be separated by disjoint open neighbourhoods. Ščepin proved that any dense subspace of the product of any family of metrizable spaces is $\kappa$-normal [10].

Let $Y$ be a subspace of $X$. A subset $A$ of $X$ is concentrated on $Y$ 2 if $A$ is contained in the closure of $A \cap Y$ in $X$. We say that $X$ is normal on $Y$ if every two disjoint closed subsets of $X$ concentrated on $Y$ can be separated by disjoint open neighbourhoods in $X$ [2].

A space $X$ is called densely normal if there exists a dense subspace $Y$ of $X$ such that $X$ is normal on $Y$ [2]. It is easy to see that every densely normal space is $\kappa$-normal. On the other hand, the converse is not true, as was shown in 8 .

In general, given a space $X$ and its dense subspace $Y$, it is not easy to determine whether $X$ is normal on $Y$, or not. Of course, if $X$ itself is normal, then $X$ is normal on every dense subspace $Y$ of $X$. But what if $X$ is not normal? Does normality of $Y$ imply that $X$ is normal on $Y$ ? How do we find out if there exists a dense subspace $Y$ of $X$ such that $X$ is normal on $Y$ ? How do we recognize such a subspace $Y$ ? The answers to these questions are not at all obvious; the only immediately clear general necessary condition for dense normality of $X$ remains $\kappa$ normality. So a natural general question to consider can be formulated as follows: which $\kappa$-normal non-normal spaces are densely normal? Here is a useful, though not difficult, result in this direction.

Proposition 2.1. Let $Y$ be a normal subspace of $X$ such that $Y$ is $C^{0}$-embedded in $X$ (that is, every bounded real-valued continuous function on $Y$ can be extended to a continuous real-valued function on $X)$. Then $X$ is normal on $Y$.

Proof. Let $A$ and $B$ be non-empty disjoint closed subsets of $X$ concentrated on $Y$. Put $A_{1}=A \cap Y$ and $B_{1}=B \cap Y$. Then $A_{1}$ and $B_{1}$ are disjoint closed subsets of $Y$ and, since $Y$ is normal, there exists a bounded continuous real-valued function $f$ on $Y$ such that $f\left(A_{1}\right)=\{0\}$ and $f\left(B_{1}\right)=\{1\}$. We can extend $f$ to a continuous real-valued function $f^{*}$ on $X$. Since $A_{1}$ is dense in $A$, it follows that $f^{*}(x)=0$, for each $x \in A$. Similarly, $f^{*}(x)=1$, for each $x \in B$. Then $U=\left\{x \in X: f^{*}(x)<1 / 2\right\}$ and $V=\left\{x \in X: f^{*}(x)>1 / 2\right\}$ are disjoint open neighbourhoods of the sets $A$ and $B$ in $X$. Thus, $X$ is normal on $Y$.

Corollary 2.2. If $X$ is a space such that there exists a dense normal subspace $Y$ of $X$ which is $C^{0}$-embedded in $X$, then $X$ is densely normal and, therefore, $\kappa$-normal.

The last two results allow us to recognize several instructive examples of densely normal spaces.

Example 2.3. Let $\tau$ be an uncountable cardinal number. We are going to show that the space $R^{\tau}$, which is well known to be $\kappa$-normal 10 and not normal (see [7]), is densely normal. Let $Y$ be the $\Sigma$-product of $\tau$ copies of $R$. Then $Y$ is a dense normal subspace of $R^{\tau}$ 7], $C^{0}$-embedded in $R^{\tau}$. Therefore, by Proposition 2.1, $R^{\tau}$ is normal on $Y$, which implies that $R^{\tau}$ is densely normal. 
Example 2.4. Let $Y$ be any normal space, $\beta Y$ the Stone-Čech compactification of $Y$, and $X$ a subspace of $\beta Y$ such that $Y \subset X \subset \beta Y$. Then $X$ is normal on $Y$; this directly follows from Corollary 2.2. In particular, every subspace $X$ of the StoneČech compactification $\beta \omega$ of the discrete space $\omega$ of natural numbers is normal on $\omega$ and, therefore, is densely normal.

The definition of dense normality, as well as Examples 2.3 and 2.4, give rise to another natural question: if $Y$ is a particularly nice dense subspace of $X$, must $X$ be densely normal on $Y$ ? For example, what is the answer if $Y$ is metrizable or even discrete? Here are some examples, clarifying the situation.

Example 2.5. Let $X$ be a dense-in-itself non-normal space, $A, B$ closed disjoint subsets of $X$, each of which is dense-in-itself, such that $A$ and $B$ cannot be separated by disjoint open neighbourhoods in $X$, and $X^{*}$ the Alexandroff double of $X$, that is, $X^{*}=(X \times\{0\}) \cup(X \times\{1\})$, where all points of $X \times\{1\}$ are isolated in $X^{*}$ and basic open sets intersecting $X \times\{0\}$ are of the form $(U \times\{0,1\}) \backslash K$ where $U$ is any non-empty open subset of the space $X$, and $K$ is any finite set.

Then $A \times\{0,1\}$ and $B \times\{0,1\}$ are canonical closed sets in the space $X^{*}$ which cannot be separated by disjoint open sets in $X^{*}$. Indeed, the sets $A_{1}=A \times\{1\}$ and $B_{1}=B \times\{1\}$ are open in $X^{*}$ and, since the spaces $A$ and $B$ do not have isolated points, $\overline{A_{1}}=A \times\{0,1\}$ and $\overline{B_{1}}=B \times\{0,1\}$. Notice that the sets $A \times\{0\}$ and $B \times\{0\}$ cannot be separated in $X^{*}$ by disjoint open sets for obvious reasons.

Therefore, the space $X^{*}$ is not $\kappa$-normal, hence, it is not densely normal. Now notice that $Y=X \times\{1\}$ is a dense subspace of $X^{*}$. It follows that $X^{*}$ is not normal on $Y$. Since $Y$ is discrete, it is metrizable. Thus, $X$ may contain a dense metrizable subspace without being densely normal or even $\kappa$-normal. This shows that the restrictions imposed on $X$ and $Y$ in Proposition 2.1 and Corollary 2.2 are not superfluous.

Can one have a space such as in Example 2.5 with a dense separable metrizable subspace? The answer is "yes", though the construction of it is slightly more involved. In fact, from the argument in Example 2.5 it is easy to see that all we need to begin with is a separable dense-in-itself space $X$ with two disjoint closed separable dense-in-itself subsets $A$ and $B$ such that $A$ and $B$ cannot be separated by open neighbourhoods in $X$. Probably, there are many ways to construct such $X$. Here is how we do it.

Example 2.6. Let $P$ be the Niemytzki half-plane, $l$ the bottom line of it (which is a closed discrete subspace of $P$ ), and $X$ a countable dense subspace of $P$ such that $X \cap l=\emptyset$ (for example, we can take $X$ to be the set of rational points of $P$ with the positive ordinate).

Now we construct a new space (consisting of three Niemytzki-like "pages") as follows. Put $Y=X \times\{0\}, Z=X \times\{1\}$, and $T=l \cup X \cup Y \cup Z$. Observe that the sets $l, X, Y$, and $Z$ are pairwise disjoint, and the set $M=X \cup Y \cup Z$ is countable.

Let us define a topology on the set $T$. All points of $M$ are declared to be isolated in $T$. Now if $x \in l$ and $\epsilon$ is a positive number, then $O_{\epsilon}(x)$ is the interior of the circle in $P$ of radius $\epsilon$ tangent to $l$ at $x, V_{\epsilon}(x)=\{x\} \cup O_{\epsilon}(x) \cup O_{\epsilon}(x) \times\{0\}$, and $W_{\epsilon}(x)=\{x\} \cup O_{\epsilon}(x) \cup O_{\epsilon}(x) \times\{1\}$.

The same argument which shows that the usual Niemytzki plane is not normal provides us with a subset $A$ of $l$ such that $A$ and $B=l \backslash A$ cannot be separated by open neighbourhoods in $P$. We fix a set $A$ with this property. For any $x \in A$, let 
$\left\{V_{\epsilon}(x): \epsilon>0\right\}$ be the standard base of $T$ at $x$. Similarly, for any $x \in B=l \backslash A$, let $\left\{W_{\epsilon}(x): \epsilon>0\right\}$ be the standard base of $T$ at $x$.

The space $T$ defined in this way is zero-dimensional, $T_{1}$ and therefore, Tychonoff. It contains a dense separable metrizable subspace $M$ (which is, in fact, a countable discrete subspace open in $T$ ). Clearly, $Y$ is an open subset of $T$ and the closure of $Y$ in $T$ is the canonical set $A^{*}=Y \cup A$. Similarly, $Z$ is an open subset of $T$ and the closure of $Z$ in $T$ is the canonical set $B^{*}=Z \cup B$. The sets $A^{*}$ and $B^{*}$ are obviously disjoint. They cannot be separated in $T$ by disjoint open neighbourhoods, since $A$ and $B$ cannot be separated by disjoint open neighbourhoods in the subspace $l \cup X$ of $T$ (exactly for the same reason, for which they cannot be separated in $P)$. Therefore, $T$ is not $\kappa$-normal and not densely normal on the countable discrete subspace $M$. Notice that $T$ is first countable. Furthermore, $T$ is obviously a Moore space (this is proved in the same way as in the case of the standard Niemytzki plane).

In the example above the larger space $T$ is not $\kappa$-normal. Now we will apply the space $T$ constructed in Example 2.6 to establish the following curious fact (notice that from Example 2.3 we know that the space $R^{c}$ is densely normal):

Theorem 2.7. There exists a countable dense subspace $H$ of the space $R^{c}$ such that $R^{c}$ is not normal on $H$.

Proof. Let us take the space $T$ constructed in Example 2.6. Obviously, $T$ can be mapped by a one-to-one continuous mapping into a separable metrizable space $L$, obtained when we identify three copies of the usual Euclidean half-plane along the border line $l$. Therefore, $T$ is (hereditarily) realcompact (see [7]). The space $T$ is also separable. Now we need the following fact:

Lemma 2.8. Every separable realcompact space $X$ is homeomorphic to a closed subspace of $R^{c}$.

Proof. Put $Y=C_{p}(X)$. Then $|Y| \leq 2^{\omega}=c$. Let $\psi$ be the canonical evaluation mapping of $X$ into $R^{Y}$. Then (see Propositions 0.5.9' and 0.5.11 in [1) $\psi(X)$ is a topological copy of $X$ closed in $R^{Y}$. Since $|Y| \leq c$, Lemma 2.8 is proved.

By Lemma 2.8, T can be represented as a closed subspace of $R^{c}$. Now, $R^{c}$ is also separable 7], therefore we can fix a countable dense subset $S$ in $R^{c}$. Since $M$ is a countable dense subset of $T, S_{1}=(S \backslash T) \cup M$ is a countable dense subspace of $R^{c}$. Let us show that $R^{c}$ is not normal on $S_{1}$. Indeed, since $M \subset S_{1}$, the sets $A^{*}$ and $B^{*}$ are concentrated on $S_{1}$. They are closed in $R^{c}$, since $T$ is closed in $R^{c}$. Obviously, $A^{*}$ and $B^{*}$ cannot be separated by open neighbourhoods in $R^{c}$, since they cannot be so separated in the smaller space $T$. Therefore, $R^{c}$ is not normal on the countable dense subspace $S_{1}$. On the other hand, we know that $R^{c}$ is $\kappa$-normal and even densely normal (see Example 2.3).

In connection with Theorem 2.7 we mention the next simple result:

Proposition 2.9. Let $X$ be a space in which the set $Y$ of all isolated points is dense. Then the following conditions are equivalent:

1) $X$ is к-normal;

2) $X$ is normal on $Y$;

3) $X$ is densely normal. 
Proof. It is enough to establish that 1) implies 2), since other implications clearly hold.

So suppose that $X$ is $\kappa$-normal, and let $A$ be any closed set in $X$ concentrated on $Y$. Our goal will be achieved if we show that $A$ is a canonical closed set in $X$. But this is so indeed, since $A=\overline{A \cap Y}$, where the set $A \cap Y$ is open in $X$, since $Y$ is an open discrete subspace of $X$.

\section{Dense normality and Relative countable compactness}

Since every normal pseudocompact space is countably compact, it is natural to consider the next question:

Question 2. Is it true that every densely normal pseudocompact space $X$ contains a dense subspace $Y$ such that $Y$ is countably compact in $X$ ? I do not know the answer to Question 2, but a partial result is available.

Theorem 3.1. If $X$ is a locally connected pseudocompact space, and $Y$ a dense subspace of $X$ such that $X$ is normal on $Y$, then $Y$ is countably compact in $X$.

Proof. We need two lemmas.

Lemma 3.2. Let $X$ be a locally connected space, $\gamma$ a disjoint family of open subsets of $X$ such that $\gamma$ is discrete in the space $Y=\bigcup\{\bar{V}: V \in \gamma\}, Z$ the closure of $\bigcup \gamma$ in $X$, and $H$ the set of accumulation points of $\gamma$ in $X$. Then $H \subset \overline{X \backslash Z}$.

Proof of Lemma 3.2. Assume the contrary. Then there exist a point $a \in H$ and an open connected subset $U$ of $X$ such that $a \in U \subset Z$. Obviously, $H=Z \backslash Y$, and $U \cap V \neq \emptyset$, for some $V \in \gamma$. By the restriction on $\gamma, \bar{V}$ is open in $Y$. Since $H$ is closed in $X$, the set $\bar{V}$ is open in $Z$. It follows that $U \cap \bar{V}$ is a proper non-empty open and closed subset of $U$, since $a \in U \backslash \bar{V}$. This is a contradiction, since $U$ is connected.

Lemma 3.3. Let $X$ be a locally connected space, and $Y$ a dense subspace of $X$ such that $X$ is normal on $Y$. Further, let $A$ be a countable discrete subspace of $Y$ such that $A$ is closed in $X$. Then for every open (in $X$ ) neighbourhood $W$ of $A$ there exists an open subset $W_{1}$ of $X$ such that $A \subset W_{1}$ and the closure of $W_{1}$ in $X$ is contained in $W$.

Proof of Lemma 3.3. Let $A=\left\{a_{n}: n \in \omega\right\}$, where $a_{n} \neq a_{m}$, when $n \neq m$. Since $X$ is regular, we can define by recursion a disjoint family $\gamma=\left\{U_{i}: i \in \omega\right\}$ of open sets in $X$ such that $a_{n} \in U_{n}$, for each $n \in \omega$, and $\bigcup\left\{U_{n}: n \in \omega\right\} \subset W$. Now we can replace each $U_{n}$ by an open neighbourhood $V_{n}$ of $a_{n}$ such that $\overline{V_{n}} \subset U_{n}$.

The family $\gamma=\left\{V_{i}: i \in \omega\right\}$ satisfies the restrictions in Lemma 3.2. Therefore, the set $H$ of all accumulation points of $\gamma$ in $X$ is contained in the closure (in $X$ ) of the set $X \backslash Z$, where $Z$ is the closure (in $X$ ) of $\bigcup \gamma$. Let $B$ be the closure of $(X \backslash Z) \cap Y$ in $X$. Clearly, $B$ is a closed set in $X$ concentrated on $Y$. Since $X \backslash Z$ is open in $X$, and $Y$ is dense in $X$, it follows that $B=\overline{X \backslash Z}$. Therefore, $H \subset B$. Notice that $B \cap(\bigcup \gamma)=\emptyset$, since $\bigcup \gamma$ is open and does not meet $X \backslash Z$.

The set $A$ is also closed in $X$ and concentrated on $Y$. Since $A \subset \bigcup \gamma \subset Z$ and $B \cap(\bigcup \gamma)=\emptyset$, it follows that $A$ and $B$ are disjoint. Since $X$ is normal on $Y$, there are disjoint open subsets $G$ and $W_{1}$ of $X$ such that $A \subset W_{1}$ and $B \subset G$. Then the closure of $W_{1}$ in $X$ does not meet $G$ and therefore, no point of $H$ is in the closure of $W_{1}$. Hence, $\overline{W_{1}} \subset \bigcup\left\{\overline{V_{i}}: i \in \omega\right\} \subset W$. Lemma 3.3 is proved. 
Now we have all we need to prove Theorem 3.1. Assume that $Y$ is not countably compact in $X$. Then we can fix $A=\left\{a_{n}: n \in \omega\right\} \subset Y$, where $a_{n} \neq a_{m}$ for $n \neq m$, such that the set $A$ is closed and discrete in $X$. Clearly, there exists a sequence $\gamma=\left\{V_{n}: n \in \omega\right\}$ of disjoint open sets in $X$ such that $a_{n} \in V_{n}$, for each $n \in \omega$.

Put $W=\bigcup \gamma$. By Lemma 3.3, there exists an open set $U$ in $X$ such that $A \subset U \subset \bar{U} \subset W$, where the closure is taken in $X$. Let $U_{n}=V_{n} \cap U$, for each $n \in \omega$. It is then a standard fact, easily verified, that $\left\{U_{n}: n \in \omega\right\}$ is a discrete family of non-empty open sets in $X$, a contradiction, since $X$ is pseudocompact.

Corollary 3.4. If $X$ is a pseudocompact, locally connected, and densely normal space, then there exists a dense subspace $Y$ in $X$ such that $Y$ is countably compact in $X$.

It is natural to ask whether Corollary 3.4 remains true for $\kappa$-normal pseudocompact locally connected spaces. The answer is 'no'.

Example 3.5. D. B. Shakhmatov constructed in [9] an infinite pseudocompact space $X$ such that every countable subset $A$ of $X$ is closed in $X$, discrete, and $b$-embedded in $X$, which means that every bounded real-valued function on $A$ can be extended to a continuous real-valued function on $X$. Another space with these properties was later described in [3]. Let $Z$ be the subspace of $C_{p}(X)$ consisting of functions with values in $I=[0,1]$. It is not difficult to show that the restrictions on $X$ imply that $Z$ is pseudocompact (see Example 1.2.5 in [1]). Clearly, $Z$ is a dense subspace of the Tychonoff cube $I^{X}$, therefore, $Z$ is $\kappa$-normal [10]. The space $Z$ is also locally connected, since it is obviously locally pathwise connected.

Let us show that $Z$ is not densely normal. By Corollary 3.4, it is enough to check that no dense subspace of $Z$ is countably compact in $Z$. Assume the contrary, and fix a dense subspace $Y$ of $Z$ such that $Y$ is countably compact in $Z$. Since $X$ is pseudocompact, it follows from Theorem 3.4.9 in [1] that the closure of $Y$ in $C_{p}(X)$ is compact. But the closure of $Y$ in $C_{p}(X)$ is $Z$, since $Z$ is closed in $C_{p}(X)$ and $Y$ is dense in $Z$. Therefore, $Z$ is compact. Since $Z$ is dense in $I^{X}$, it follows that $Z=I^{X}$, that is, every mapping of $X$ into $I$ is continuous. But this can be the case only if the space $X$ is discrete, a contradiction, since $X$ is infinite and pseudocompact.

Thus, $X$ is a locally connected, pseudocompact, $\kappa$-normal, not densely normal space.

Notice that the results above motivate the next question:

Question 3. Is there a countably compact $\kappa$-normal space $X$ such that $X$ is not densely normal? What if, in addition, we assume $X$ to be locally connected?

The next particular case of Question 3 seems to be especially interesting. We recall that every countably compact topological group is $\kappa$-normal (see [10]).

Question 4. Is every countably compact topological group densely normal?

\section{ON DENSE DENSELY NORMAL SUBSPACES}

In this section we have another look at the main result of section 1, taking the approach developed in sections 2 and 3 . Indeed, at this point it is only very natural to ask whether there exists a Tychonoff space $X$ such that no dense subspace of $X$ is densely normal. Is $C_{p}\left(\omega_{1}+1\right)$ such a space? Below we show that the answer to this question is in the affirmative. The proof follows the same lines as the main 
argument in section 1 , but we have to use suitable generalizations of the results in $C_{p}$-theory we relied upon in section 1 . We formulate the theorems we need; they are just relative versions of the "absolute" results. Luckily, not only they remain true, but their proofs can be written down as straightforward adaptations of the (already published) proofs of the "absolute" counterparts, so that we can omit the proofs.

Recall that subsets $A$ and $B$ of a space $X$ are said to be separated (in $X$ ) if $\bar{A} \cap B=\bar{B} \cap A=\emptyset$. Let $Z$ be a subspace of $Y$. We say that the extent of $Z$ in $Y$ is countable (and write $e(Z, Y) \leq \omega$ ) if every closed in $Y$ discrete subspace of $Z$ is countable. If every open covering of $Y$ contains a countable subfamily covering $Z$, we say that the Lindelof number of $Z$ in $Y$ is countable and write $l(Z, Y) \leq \omega$. From these definitions it is clear how the (relative) cardinal invariants $e(Z, X)$ and $l(Z, Y)$ are defined in the general case (see 2$]$ ).

Here are the main results of this section:

Theorem 4.1. Let $X=C_{p}\left(\omega_{1}+1\right), Y$ a dense subspace of $X$, and $Z$ a dense subspace of $Y$. Then $Y$ is not normal on $Z$.

Corollary 4.2. No dense subspace of $C_{p}\left(\omega_{1}+1\right)$ is densely normal.

To prove Theorem 4.1 we need the following assertions, the first of which is well known (see, for example, [1, Lemma 1.6.1).

Lemma 4.3. Let $M$ be a separable space, $L$ a set, and $U, V$ disjoint open subsets of the product space $M^{L}$. Then there exists a countable subset $S$ of $L$ such that the sets $\pi_{S}(U)$ and $\pi_{S}(V)$ are disjoint (where $\pi_{S}$ is the natural projection of $M^{L}$ onto $\left.M^{S}\right)$ and separated (in $\left.M^{S}\right)$.

Proposition 4.4. Let $Y$ be a dense subspace of the product $M^{\omega_{1}}$ of $\omega_{1}$ copies of a separable space $M$, and $Z$ a dense subspace of $Y$ such that $Y$ is normal on $Z$. Then the extent of $Z$ in $Y$ is countable, that is, $e(Z, Y) \leq \omega$.

To prove Proposition 4.4, we only have to introduce some obvious changes in the proof of Theorem 1 in [6]. Lemma 4.3 plays the key role in the proof of Proposition 4.4. Proposition 4.4 works effectively in combination with the next result from 3 (see Corollary 8.20):

Lemma 4.5. If a subspace $Z$ of $C_{p}\left(\omega_{1}+1\right)$ separates points of $\omega_{1}+1$, then there exists an uncountable discrete subspace $A$ of $Z$ such that $A$ is closed in $C_{p}\left(\omega_{1}+1\right)$.

Since every dense subspace $Z$ of $C_{p}\left(\omega_{1}+1\right)$ separates points of $\omega_{1}+1$, Theorem 4.1 follows from Proposition 4.4 and Lemma 4.5.

Similarly, we can strengthen Theorem 1.4:

Theorem 4.6. There exists a submetrizable space $X$ with countable Souslin number such that no dense subspace of $X$ is densely normal.

Proof. We take the same spaces $K$ and $X$ as in the proof of Theorem 1.4: $K=$ $A \cup\left(\omega_{1}+1\right)$ (where $A$ is a countable dense subset of $K$ ) and $X=C_{p}(K)$. Notice that $K$ is $\sigma$-compact and, therefore, a Lindelöf $\Sigma$-space. Now to complete the proof of Theorem 4.6 we argue exactly as in the proofs of Theorems 1.4 and 4.1. 


\section{REFERENCES}

[1] A.V. Arhangel'skii, Topological function spaces, Kluwer Academic Publishers, 1992. MR 92i:54022

[2] A.V. Arhangel'skii, Relative topological properties and relative topological spaces, Topology Appl. 70 (1996), 87-99. MR 97f:54030

[3] A.V. Arhangel'skii, $C_{p}$-theory. In: Recent progress in General Topology, pages 1-48, NorthHolland, Amsterdam, 1992. MR 95g:54004

[4] Arhangel'skii A.V. and V.V. Uspenskij, On the cardinality of Lindelöf subspaces of function spaces, Comment. Math. Univ. Carolinae 27:4 (1986) 673-676. MR 88d:54003

[5] Baturov D.P., Subspaces of function spaces, Vestnik. Moskov. Univ. Ser. Mat. Mech. 4 (1987) 66-69. MR 89a:54018

[6] Baturov D.P., Normality in dense subspaces of products, Topology and Appl. 36 (1990) 111116. MR 91j:54033

[7] R. Engelking, General Topology, Heldermann Verlag, Berlin, 1989. MR 91c:54001

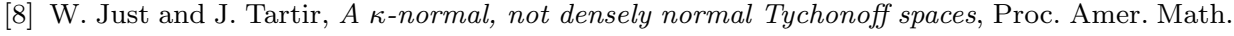
Soc. 127 (1999), no. 3, 901-905. MR 99f:54028

[9] D.B. Shakhmatov, A pseudocompact Tychonoff space all countable subsets of which are closed and $C^{*}$-embedded, Topology Appl. 22 (1986), 139-144. MR 87h:54009

[10] E.V. Ščepin, Real-valued functions and spaces close to normal, Sib. Matem. Journ. 13:5 (1972), 1182-1196.

Department of Mathematics, 321 Morton Hall, Ohio University, Athens, Ohio 45701

E-mail address, January 1-June 30: arhangel@bing.math.ohiou.edu

E-mail address, July 1-December 31: arhala@arhala.mccme.ru 